\title{
Minimum Overlapping Layers and Its Variant for Prolonging Network Lifetime in PMRC-based Wireless Sensor Networks
}

\author{
Qiaoqin $\mathrm{Li}^{12}$, Mei Yang ${ }^{1}$, Hongyan Wang ${ }^{1}$, Yingtao Jiang ${ }^{1}$, Jiazhi Zeng ${ }^{2}$ \\ ${ }^{1}$ Department of Electrical and Computer Engineering, University of Nevada, Las Vegas, NV, 89154 \\ ${ }^{2}$ College of Computer Science and Technology, University of Electronic Science and Technology, Chengdu, China \\ Emails: ${ }^{1}$ meiyang@egr.unlv.edu, wangh7@unlv.nevada.edu, yingtao@egr.unlv.edu\}, \\ ${ }^{2}\{$ helenli803@163.com,jzzeng@uestc.edu.cn\}
}

\begin{abstract}
The Overlapping Layers (OL) scheme proposed in our previous work provides a solution to balance the load of cluster heads at different layers in the PMRC-based wireless sensor networks. However, in the OL scheme, the layer boundary and the overlap range are static through the network lifetime. The network lifetime is still limited by some nodes which have only one candidate cluster head. To overcome this limitation, in this paper, we propose the Minimum Overlapping Layers (MOL) scheme with gradually changed layer boundary through network lifetime and its variant, the MOL with Initial Overlap (MOLIO) scheme. The simulation results of the OL, MOL, and MOLIO schemes show that the MOL scheme significantly prolongs the network lifetime than the OL scheme for most transmission ranges and the MOLIO scheme achieves better results than the MOL scheme at larger transmission ranges.
\end{abstract}

Index Terms-Wireless sensor networks, overlapping layers, PMRC

\section{INTRODUCTION}

Due to the benefits of low cost, rapid deployment, selforganization capability and cooperative data processing, wireless sensor networks (WSNs) have been proposed as a practical solution for a wide range of applications [1], such as military surveillance and habitat monitoring, etc. Since the power supply for each sensor node is usually extremely limited, energy efficiency is the primary challenge of WSNs. Previous research shows that clustered structure [3] and multi-hop routing [1][2] achieve better energy efficiency for large scale wireless sensor networks.

In [8], a highly scalable network architecture, named as Progressive Multi-hop Rotational Clustered (PMRC) structure is proposed for constructing large scale WSNs. In the PMRC structure, sensor nodes are partitioned into layers according to their distance to the sink node. A cluster is composed of nodes located in the same layer and cluster head in the upper layer closer to the sink node. The cluster head is responsible for forwarding data to its upstream layer. As traffic load is concentrated on the cluster heads closer to the sink node, these cluster heads will deplete their power faster, which limit the lifetime of the whole network. This is referred as the bottleneck problem of the PMRC-based WSN.

In the literature, several schemes have been proposed to solve the bottleneck problem in WSNs. In [6], an Unequal Clustering (UCS) modeling is proposed to balance energy consumption of cluster heads in multi-hop WSNs. This work is focused on a heterogeneous network where cluster heads are deterministically deployed at some pre-computed locations, thus it's easy to control the cluster size. In [4], an Energy-Efficient Unequal Clustering (EEUC) mechanism is proposed to partition the sensor nodes into unequal size such that clusters closer to the sink node are expected to have smaller cluster size. Thus these clusters will consume lower energy during the intra-cluster data processing, and can preserve more energy for the inter-cluster relay traffic. They also propose an energy-aware multi-hop routing protocol for the inter-cluster communication. A similar problem of unbalanced energy consumption among cluster heads also exists in single-hop sensor networks. The Energy Efficient Clustering Scheme (EECS) [9] is proposed to produce clusters of unequal size in single-hop networks.

To solve the bottleneck problem in PMRC-based WSNs, the Overlapping Layers (OL) scheme is proposed in [7] to balance the relay load at the cluster heads for all layers and prolong network lifetime. However, in the OL scheme, the layer boundary and the overlapping range are static during the network lifetime. The network lifetime is still limited by some nodes which have only one candidate cluster head.

To overcome this problem, in this paper, we propose the Minimum Overlapping neighbor Layers (MOL) scheme with gradually changed layer boundary through network lifetime to achieve load balance and energy efficiency in the whole network area. Then we propose the MOL with Initial Overlap (MOLIO) scheme to further improve the MOL scheme at larger transmission ranges.

The rest of this paper is organized as follows. Section 2 describes the network model, OL scheme, and energy model. Section 3 describes the details of the MOL and MOLIO schemes. Simulation results are presented in Section 4. Section 5 concludes the paper.

\section{PRELIMINARIES}

\section{A. Network Model and OL Scheme}

Without loss of generality, assume that all the sensor nodes are homogeneous and have the same capability. Assume that all the sensor nodes are active in transmission and a portion of these nodes are active in sensing data. As described before, in the PMRC structure [8], each sensing node transmits its sensed data to its cluster head. Each cluster head sends the sensed data within its cluster and forwards data coming from the cluster heads in its downstream layer to the cluster head in its upstream layer. 
As discussed in [8], in the PMRC structure, two cluster head selection strategies can be used: selecting single cluster head or double cluster heads for each cluster. Once the selected cluster head(s) of a cluster are exhausted, the sensor nodes in the cluster will send a reelection request to other candidate cluster heads which will forward the request to the sink. Once receiving a reelection request, the sink will initiate the network recreation process. If there is one sensor node that cannot find any candidate cluster head, the network is considered as partitioned.

Similar to other multi-hop structures, the PMRC structure also suffers from the bottleneck problem. Particularly, the cluster heads closer to the sink node are burdened with heavy relay traffic and tend to die early, which reduces network coverage and causes network partition.

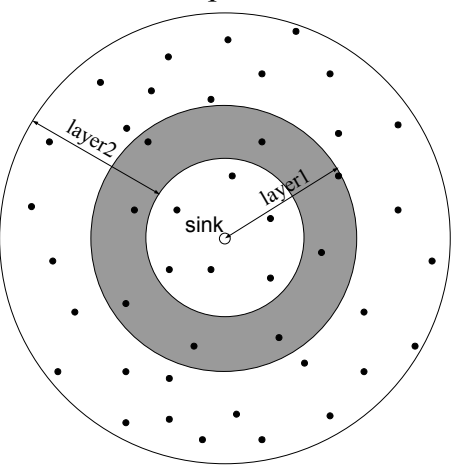

Fig. 1 OL scheme for PMRC-based sensor network.

To solve this problem, the OL scheme is proposed to balance the relay load at the cluster heads for all layers [7]. Fig. 1 illustrates an OL-enabled PMRC-based sensor network deployed in a circular area with the sink node located at the center of the area. As shown in the figure, layer 1 occupies a circular area and layer 2 is shown in a ring shape. The grey area indicates the overlapped area for layer 1 and layer 2 . Note that the sensor nodes in the grey area still belong to layer 1 while they are the candidate cluster heads for clusters in layer 2. By this way, more replacing cluster heads can be found from these candidate nodes. In addition, by overlapping layers, the size of the clusters formed in layer 2 tends to be smaller, which will save the energy consumed in intra-cluster communication. Consequently, the network lifetime can be prolonged.

When more than two layers exist in the network, the overlapping between other adjacent layers is also needed. In [7], analysis is performed to determine the desirable overlapped ranges between neighbor layers. Simulation results show that the scenarios with overlapped layers outperform the scenarios without overlapped layers significantly in terms of network lifetime.

\section{B. Energy Model}

In our model, we only consider the energy consumption in transmission and reception, which dominates the overall energy consumption of each node [3]. Assume that only the free space channel model is used. The energy consumed for transmitting an $l$-bit packet over a distance of $d$ is:

$$
\begin{aligned}
E_{t x}(l, d)=E_{t x-\text { elec }}(l) & +E_{t x-\text { amp }}(l, d) \\
& =l\left(E_{\text {elec }}+\varepsilon_{f s} d^{2}\right),
\end{aligned}
$$

and the correspongding energy consumed in data receiving is:

$$
E_{r x}(l)=E_{r x-\text { elec }}(l)=l E_{\text {elec }} \text {. }
$$

In this paper, the system parameters $E_{\text {elec }}$ and $\varepsilon_{f s}$ are set as: $E_{\text {elec }}=50 \mathrm{~nJ} / \mathrm{bit}$, and $\varepsilon_{f s}=10 \mathrm{pJ} / \mathrm{bit} / \mathrm{m}^{2}$.

For each sensor node, the total energy consumed during its lifetime is:

$$
E_{\text {total }}=E_{\text {data }}+E_{c t l}
$$

where $E_{\text {data }}$ and $E_{c t l}$ represent energy consumed for sending/receiving data packets and control packets, respectively. And

$$
E_{c t l}=E_{\text {ctl-topology }}+E_{\text {ctl-beacon }} .
$$

$E_{\text {ctl-topology }}$ represents the energy consumed for the control packets used for network recreation, which is determinded by the number of network recreations and the number of layers generated in each recreation. $E_{\text {ctl-beacon }}$ represents the energy consumed by beacon packets.

To achieve energy efficiency, $E_{c t l}$ shall be minimized as much as possible. $E_{\text {ctl-beacon }}$ in our model is reduced in the following way. A sensor node only sends beacon to notify other nodes when its energy drops below a threshold. All nodes in the transmission range should process the beacon packet and determine if the reelection request should be generated.

\section{MINIMUM OVERLAPPING LAYERS AND ITS VARIANT}

\section{A. The MOL scheme}

In the OL scheme, the layer boundary and the overlap range are static during the network lifetime. It is observed that the network lifetime is still limited by some nodes which have only one candidate cluster head. To overcome this limitation, we propose the Minimum Overlapping neighbor Layers scheme with gradually changed layer boundary through network lifetime to achieve better load balance and energy efficiency in the whole network area.

Without loss of generality, we assume that a node is eligible to be elected as a cluster head only if its residue energy is higher than a pre-defined energy threshold. In the OL scheme, an overlap between two neighbor layers is not necessary if the cluster head has its residue energy higher than the threshold. As such, the initial overlapped area between neighbor layers in the OL scheme may be reduced to consist of only the initial cluster head of a cluster. When the residue energy of the initial cluster head falls below the energy threshold, it will be deliberately "pushed" to its downstream layer by increasing its layer number by one. The result is that the overlap between these two neighbor layers may grow a little larger towards the upstream layer direction on the next round of network recreation. Consequently, throughout the network lifetime, minimum overlap between any neighbor layers is kept and the cluster size is dynamically changed. 
Fig. 2 illustrates the gradually growing overlap between neighbor layers in the MOL scheme. As shown in Fig. 2(a), initially, node 2 in layer 1 is selected as the cluster head for the cluster composed of nodes 3, 4, 5, 6 in layer 2. After the residue energy of node 2 drops below the threshold, it will be "pushed" to layer 2 . We say node 2 "retires" from the cluster head position. Then the network is recreated and node 1 is selected as the head of the cluster which consists of nodes 2 , 3, 4, 5 (see Fig. 2(b)). Node 6, which is originally within the transmission range of node 2, is "pushed" to layer 3 as it is out of the transmission range of any node in layer 1. The result is that the overlapped range between layer 1 and layer 2 is growing larger towards layer 1 .

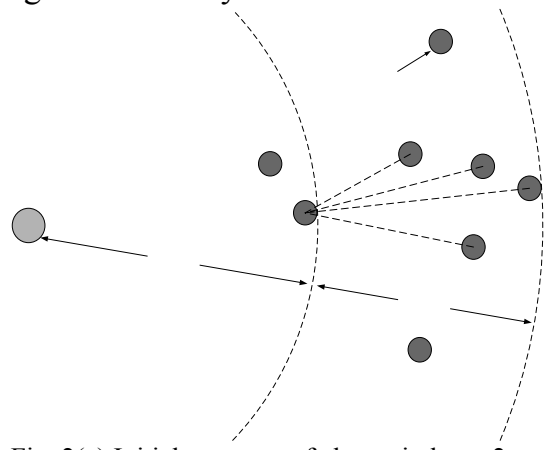

Fig. 2(a) Initial structure of cluster in layer 2.

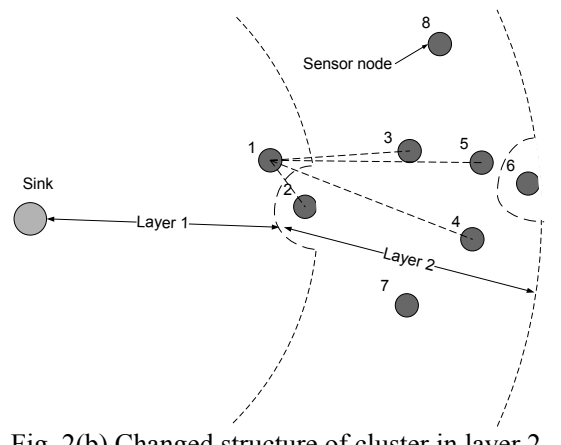

Fig. 2(b) Changed structure of cluster in layer 2.

\section{B. Properties of $M O L$}

First, different from the OL scheme, the required minimum overlap between neighbor layers is gradually increased on demand during network lifetime. Also not as in the OL scheme, neighbor layers can be irregularly overlapped and a layer can be in irregularly ring-like shape. Due to the dynamic change of layer boundary and cluster topology, the routing path from a source sensor node to the sink node is changed accordingly. However, the routing path will always have the lowest number of hop counts, which is guaranteed by the basic rule how the layers are formed in the PMRC structure.

Second, the MOL scheme overcomes the limit caused by static network topology control. As such, the MOL scheme can adapt to any randomly deployed network as long as the initial topology is connected (i.e. any sensor node has at least one neighbor node which is within its transmission range). Due to its dynamic feature, the MOL scheme provides fault tolerance against sudden failure of sensor node(s) provided that the remaining topology is still connected. Due to this property, the deployment of the network is made easier.

Third, the MOL scheme inherently helps in balancing the energy consumption among cluster heads within the same layer. Compared with the OL scheme, the MOL scheme promotes this balance in a dynamic way. In addition, the deliberate change of layer number of "retired" cluster heads will also help reducing their energy consumption in later communication.

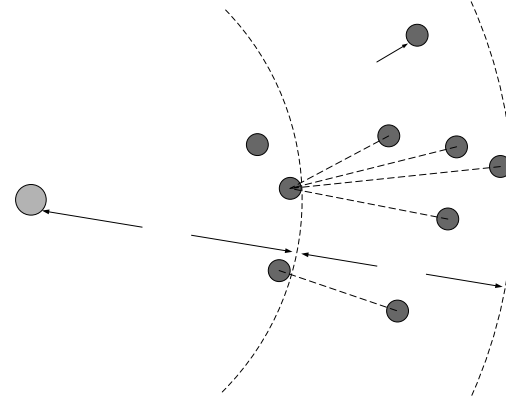

Fig. 3(a) Unbalanced energy consumption.

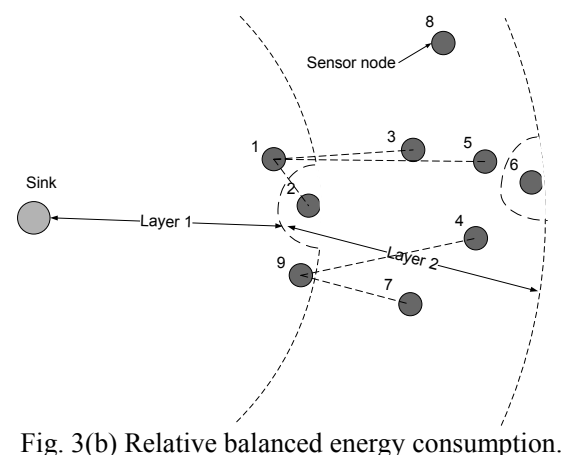

Fig. 3 illustrates an example of how energy consumption is balanced. As shown in Fig. 3(a), as the cluster head for a larger cluster, node 2 drains out its energy faster than node 9 , which is the cluster head for a smaller cluster. Fig. 3(b) shows that after network recreation, node 1 replaces node 2 as a new cluster head while node 9 still acts as a cluster head. Node 4 is switched from its original cluster to the other cluster headed by node 9 . As a result, the unequal energy consumption among different clusters of the same layer will be improved. Also, after network recreation, node 2 joins the cluster headed by node 1 , which is closer to node 2 than its original cluster head (i.e., the sink node). Communication energy needed for node 2 can be saved in the new topology.

\section{The MOLIO Scheme}

As mentioned in Section 2.1, in the OL scheme, the cluster size tends to be smaller, which helps improving the energy efficiency, especially when the transmission range is larger. Combining the advantages of both OL and MOL schemes, we propose the MOL with Initial Overlap scheme. In this scheme, all neighbor layers are initially overlapped following the analyzed overlap ranges [7]. When one node fails, the MOL scheme is used to gradually increasing the overlap range between neighbor layers. 


\section{PERFoRMAnCE EVAluATION}

To evaluate the performance of the proposed MOL scheme and its variant, simulations of OL, MOL, and MOLIO schemes have been conducted on the simulation model [7] developed on the OPNET Modeler network simulator. A simple CSMA-based channel access model is implemented as the media access scheme [5].

\section{A. Simulation Setting}

In the simulation, we assume a $200 m \times 200 m$ geographical area covered by 400 sensor nodes with the sink node located at the center. Table 1 lists some basic parameters used in the simulations.

Table 1. Simulation parameters.

\begin{tabular}{|l|l|}
\hline Parameter & Value \\
\hline Sensor field area & $200 \mathrm{~m} \times 200 \mathrm{~m}$ \\
\hline Number of nodes & 400 \\
\hline Radio transmission range & $\{20,40,60,80\} \mathrm{m}$ \\
\hline Initial energy per node & $1 \mathrm{~J}$ \\
\hline Buffer size & 1000 packets \\
\hline Channel bandwidth & $1 \mathrm{Mbps}$ \\
\hline Processing speed & $1 \mathrm{Mbps}$ \\
\hline Packet generation rate & $2 \mathrm{pkt} / \mathrm{s}$ \\
\hline Simulation time & Until network partition \\
\hline
\end{tabular}

The following performance metrics are collected.

Average packet latency: the latency of a packet is defined as the time difference between the time when the packet is generated at the source node and the time when the packet is received at the sink node. This latency includes the delay on each hop the packet traversed, which is composed of the transmission and receiving delay, the propagation delay, as well as the processing and queueing delay on each hop.

Average energy (consumption) per packet: the energy cosumption per packet is calculated over all the hops that a packet traverses, including transmission and reception energy.

Time to first node death: in our simulation, we consider only the node death due to drained energy. Generally, this metric reflects the worst lifetime.

Network lifetime: it is defined as the time when the network is partitioned or all source nodes drain out their energy.

Number of network recreation times: it is defined as the number of network recreation times.

In the following, we present the simulation results of the five performance metrics for three different scenarios: 1) OL with $x_{1}=0.5, x_{2}=0.3$, and $\left.x_{3}=0.2 ; 2\right)$ MOL; 3) MOLIO with $x_{1}=0.5, x_{2}=0.3$, and $x_{3}=0.2$. For each scenario, two types of cluster head selection strategies: single-head and doublehead are simulated. For clarity purpose, we only show the results with the single-head strategy in all figures.

All simulations are based on the same random deployment with all nodes uniformly distributed in the sensor area. A portion of sensor nodes (40 in our simulations) are selected as source nodes to generate and send data. Without loss of generality, these source nodes are randomly distributed in the sensor area. The results shown are the averaged results of five sets of 40 source nodes.

\section{B. Simulation Results}

Fig. 4-8 present the performance metrics of all three scenarios. Fig. 4 shows the average packet latency vs. transmission range. In general, the average packet latency deceases with the increase of transmission range $R_{t}$. The reason is that when $R_{t}$ increases, less layers are generated in the network and the number of hops traversed by a packet decreases, which leads to a lower latency. Fig. 4 also shows that the MOL scheme encounters much higher delay than the OL scheme when $R_{t} \leq 40 \mathrm{~m}$. Without the initial overlap, the MOL scheme experiences more network recreations (see Fig. 8 ), during which extra data delay is resulted. Due to similar reason and the impact of the initial overlap to the network topology, the MOLIO scheme has medium delay for $R_{t} \leq 40 \mathrm{~m}$.

Fig. 5 shows the average energy per packet vs. transmission range $R_{t}$. The energy consumed to transmit a packet depends on both the number of hops and the distance of each hop. The average energy per packet is decreased for $R_{t} \leq 40 m$ due to less number of hops traversed, but it is increased for $R_{t}>40 \mathrm{~m}$ as higher transmission energy is needed for larger $R_{t}$ 's. Fig. 5 also shows that under the same transmission range, the MOL scheme consumes less average energy per packet than the OL scheme in most cases as the number of hops resulted in the MOL scheme is lesser without the initial overlap.

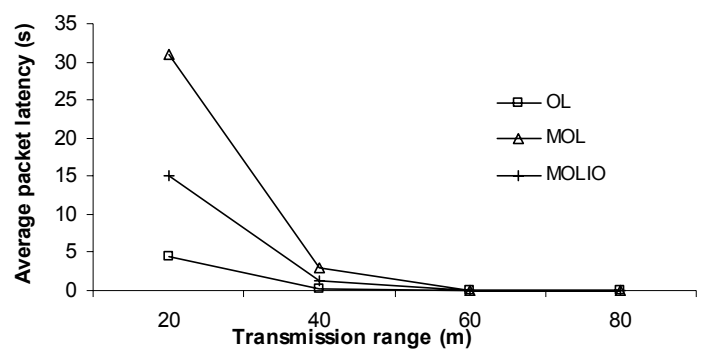

Fig. 4 Average packet lantency vs. transmission range.

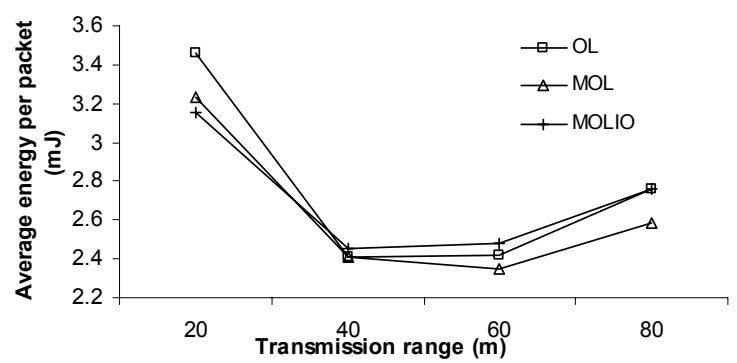

Fig. 5 Average energy per packet vs. transmission range.

Fig. 6 shows the time to first node death vs. transmission range $R_{t}$. It shows that the time to first node death decreases with the increase of $R_{t}$. Under the same transmission range, the MOLIO scheme has the same time to first node death as the OL scheme. This is because the the initial network topologies generated using the OL scheme and the MOLIO scheme are the same.

Fig. 7 presents the network lifetime vs. transmission range $R_{t}$. Generally the network lifetime for all scenarios shows an increasing trend followed by a slightly decreasing trend with $R_{t}$ increasing except the OL scheme. This can be explained 
that with the increase of transmission range, the number of candidate cluster heads for each layer increases, which helps prolonging the network lifetime. Meanwhile, the transmission energy increases with the increase of transmission range, which reduces the network lifetime. Due to the two contradicting factors, the highest network lifetime is achieved at $R_{t}=60 \mathrm{~m}$ for both MOL and MOLIO schemes. However, for the OL scheme, the layer boundary is fixed all the time. The impact of more candidate cluster heads weights more than the more energy consumption. That is why the network lifetime of the OL scheme increases all the way with $R_{t}$ increasing.

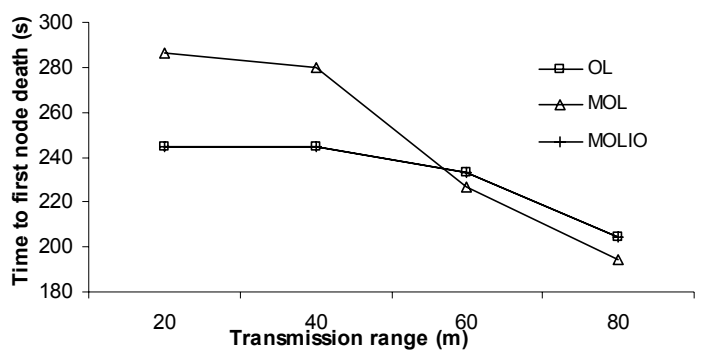

Fig. 6 Time to first node death vs. transmission range.

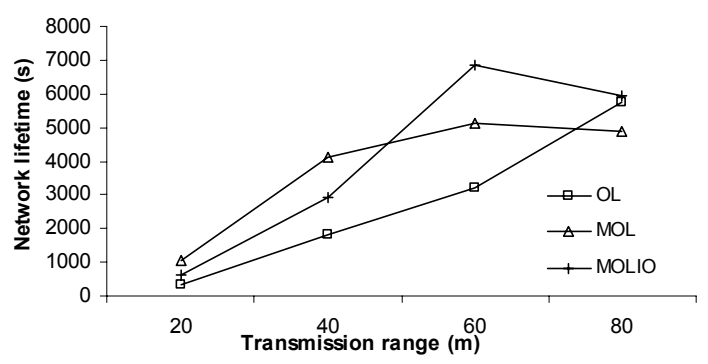

Fig. 7 Time to network partition vs. transmission range.

Fig. 7 also shows that for $R_{t} \leq 60 m$, the MOL scheme significantly prolongs the network lifetime (by the largest $230 \%$ at $R_{t}=20 \mathrm{~m}$ ) compared with the OL scheme. When $R_{t}=$ $80 \mathrm{~m}$, the network lifetime of the MOL scheme is not as good as those of the OL and MOLIO schemes. The reason is that without initial overlap, the clusters formed using the MOL scheme are much larger than the clusters formed using the OL scheme. Hence, the cluster heads and source nodes are exhuasted sooner in the MOL scheme. Actually due to the high transmission energy needed at $R_{t}=80 \mathrm{~m}$, the network lifetime of all three schemes is limited by the time when all the source nodes fail. As one can see from the figure, combing the advantages of both OL and MOL schemes, the MOLIO scheme achieves the longest network lifetime at $R_{t}=$ $60 \mathrm{~m}$. However, for $R_{t} \leq 40 \mathrm{~m}$, the network lifetime of the MOLIO scheme is not as good as that of the MOL scheme due to the impact of the intial overlap to the network topology.

Fig. 8 represents the number of network recreation times vs. transmission range $R_{t}$. Generally, the number of network recreation times shows an increasing trend followed by a decreasing trend with the increase of $R_{t}$. This can be explained that when the transmission range is getting larger, more candidate cluster heads are available, which leads to higher number of network recreation times. For MOL and MOLIO schemes, there is a drop of number of network recreation times at larger transmission ranges. This is caused by the exhaustion of all source nodes.

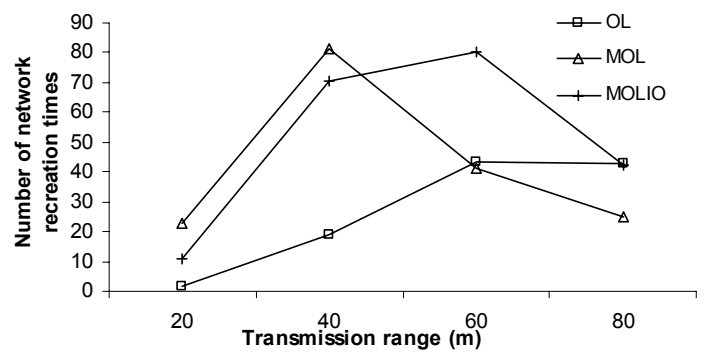

Fig. 8 Number of network recreation times vs. transmission range.

\section{CONCLUSION}

In this paper, we proposed the MOL scheme with gradually changed layer boundary through network lifetime and its variant, the MOLIO scheme, for the PMRC-based wireless sensor networks. We showed that the MOL scheme overcomes the limitation caused by static network topology control and can adapt to any randomly deployed network as long as the initial topology is connected. Simulation results of the OL, MOL, and MOLIO schemes confirmed that the MOL scheme significantly prolongs network lifetime. Combining the advantages of the OL and MOL schemes, the MOLIO scheme further improves the result of the MOL scheme at larger transmission ranges. Future work includes analysis of the network lifetime limitation with the MOL and MOLIO schemes for the PRMC-based WSNs.

\section{REFERENCES}

[1] I. F. Akyildiz, W. Su, Y. Sankarasubramaniam, and E. Cayirci, "A survey on sensor networks," IEEE Cотmu. Mag., pp. 102-114, Aug. 2002.

[2] J. Ding, K. Sivalingam, R. Kashyaoa, and L. J. Chuan, "A multi-layered architecture and protocols for large-scale wireless sensor networks," Proc. IEEE SVTC, 2003, pp. 1443-1447.

[3] W. Heinzalmen, A. Chandrakasan, and H. Balakrishman, "An application-specific protocol architecture for wireless microsensor networks," IEEE Trans. Wireless Communications, vol. 1, no. 4, pp. 660-670, 2002.

[4] C. Li, M. Ye, G. Chen, and J. Wu, "An energy-efficient unequal clustering mechanism for wireless sensor networks," Proc. 2nd IEEE Conf. Mobile Ad-hoc and Sensor Systems (MASS), 2005, pp. 8-15.

[5] Q. Li, M. Yang, H. Wang, Y. Jiang, and J. Zeng, "A finite queue model analysis of PMRC-based wireless sensor networks," Proc. Int'l Conf. Wireless Networks, 2008, pp. 30-35.

[6] S. Soro and W. Heinzelman, "Prolonging the lifetime of wireless sensor networks via unequal clustering," Proc. IEEE $19^{\text {th }}$ Int'l Parallel and Distributed Processing Symp. (IPDPS), 2005.

[7] H. Wang, M. Yang, Y. Jiang, S. Wang, and L. Gewali, "Overlapped layers for prolonging network lifetime in multi-hop wireless sensor networks," Proc. ITNG , 2008, pp. 755-760.

[8] M. Yang, S. Wang, A. Abdelal, Y. Jiang, and Y. Kim, "An improved multi-layered architecture and its rotational scheme for large-scale wireless sensor networks," Proc. IEEE CCNC, 2007, pp. 855-859.

[9] M. Ye, C. F. Li, G. H. Chen, and J. Wu, "EECS: an energy efficient clustering scheme in wireless sensor networks," Proc. IEEE Int'l Performance Computing and Communication Conf. (IPCCC), 2005, pp. 535-540. 